\title{
Time-of-flight compressed-sensing ultrafast photography for encrypted three-dimensional dynamic imaging
}

Jinyang Liang, Liang Gao, Pengfei Hai, Chiye Li, Lihong V. Wang

Jinyang Liang, Liang Gao, Pengfei Hai, Chiye Li, Lihong V. Wang, "Time-offlight compressed-sensing ultrafast photography for encrypted threedimensional dynamic imaging," Proc. SPIE 9761, Emerging Digital Micromirror Device Based Systems and Applications VIII, 97610I (15 March 2016); doi: $10.1117 / 12.2208810$

SPIE. Event: SPIE OPTO, 2016, San Francisco, California, United States 


\title{
Time-of-flight Compressed-sensing Ultrafast Photography for Encrypted Three-dimensional Dynamic Imaging
}

\author{
Jinyang Liang ${ }^{\dagger}$, Liang Gao ${ }^{\dagger}$, Pengfei Hai, Chiye Li, and Lihong V. Wang* \\ Optical Imaging Laboratory, Department of Biomedical Engineering, Washington University in St. \\ Louis, Campus Box 1097, One Brookings Drive, St Louis, Missouri 63130, USA \\ ${ }^{\dagger}$ These authors contributed equally to this work.
}

\begin{abstract}
We applied compressed ultrafast photography (CUP), a computational imaging technique, to acquire three-dimensional (3D) images. The approach unites image encryption, compression, and acquisition in a single measurement, thereby allowing efficient and secure data transmission. By leveraging the time-of-flight (ToF) information of pulsed light reflected by the object, we can reconstruct a volumetric image $(150 \mathrm{~mm} \times 150 \mathrm{~mm} \times 1050 \mathrm{~mm}, x \times y \times z)$ from a single camera snapshot. Furthermore, we demonstrated high-speed 3D videography of a moving object at 75 frames per second using the ToF-CUP camera.
\end{abstract}

Keywords: 3D imaging; Ultrafast imaging; Compressed sensing; Streak camera; Digital Micromirror Device (DMD)

\section{INTRODUCTION}

Three-dimensional (3D) imaging technologies have been extensively studied for many years, going back to the "mirror stereoscope" invented by Sir Charles Wheatstone in 1838[1]. Since then, 3D imaging techniques have been used in many applications, including remote sensing, biology, and entertainment[2-4]. In the past decade, they have also been widely employed in the safety and national security communities[5], in applications such as biometrics[6,7], under-vehicle inspection[8, 9], and battlefield evaluation[10, 11]. These applications impose demanding requirements - the 3D images must be captured and transmitted to users in a secure and fast manner.

The scattered photons from the object carry a variety of tags, such as emittance angle and time-of-flight (ToF), which convey the 3D surface information. Using these photon tags, numerous 3D imaging techniques have been developed, including structured-illumination[12, 13], holography[14], streak imaging[15, 16], integral imaging[17], multiple camera or multiple single-pixel detector photogrammetry[18, 19], and ToF detection[20]. Among these techniques, holography has demonstrated secure 3D surface imaging. Holography-based encryption is optically performed by using the diffraction pattern of a pseudo-random phase or amplitude mask[14]. This mask acts as the decryption key in reconstructing images of the 3D object from different viewing angles. However, as an interference-based approach, holographic imaging system is sensitive to motion during the relatively long exposure, which can severely degrade image quality.

ToF detection is another commonly used approach for 3D imaging. By measuring the ToF of a light signal between the object and the detector, the depth of the 3D object can be quantified. Among developed ToF detectors are the Microsoft Kinect $^{\mathrm{TM}}$ sensor[21] for Xbox One and the single photon avalanche diode detector[22]. Although having largely improved the detection sensitivity, these ToF detectors acquire 3D images through multiple measurements, which limits applications for imaging fast-moving 3D objects.

To mitigate motion distortion in 3D images, single-shot ToF detection is an attractive approach. Among developed techniques[21-27], the 3D Flash LIDAR camera[26] has gained the most attention. This device can collect the entire spatiotemporal datacube within a single camera exposure, with an imaging speed up to $30 \mathrm{~Hz}[26]$. However, the best achievable depth resolution is limited to $\sim 10 \mathrm{~cm}$ [27]. In addition, the 3D images are first recorded on the sensor, followed by data compression and encryption for transmission[28]. Therefore, the sensor itself suffers from a heavy data load. In addition, the separate recording, compressing, and encrypting processes create a security loophole in the system.

*Corresponding author: LHWANG@WUSTL.EDU; phone: 1 (314) 935-6152; 
To overcome these limitations, we present single-shot encrypted 3D imaging using compressed ultrafast photography (CUP). As a novel ultrafast imaging technique, CUP captures transient events with picosecond temporal resolution in a single snapshot[29]. Herein, we integrated CUP with active illumination and constructed a new ToF-CUP camera for snapshot volumetric imaging. Compared with previous techniques[30-33], our system has three major advantages. First, the united process incorporating the encryption of depth data with the compressed acquisition of 3D data protects the security of private content. Second, each volumetric image is stored as a $2 \mathrm{D}$ snapshot in the measurement, which significantly reduces the data acquisition load on the sensor and therefore allows faster data communication to users. Third, the ToF-CUP camera can capture 3D images with a rate up to 75 volumes per second with a $1-\mathrm{cm}$ depth resolution, outperforming the state-of-the-art single-shot 3D cameras. In the following, we will describe the principle of our approach, present the system prototype, and demonstrate encrypted 3D videography by imaging various moving 3D objects.

\section{METHOD}

\subsection{Principle}

The principle of CUP has been detailed in our recent publication[29]. Here, we provide CUP with active illumination and detect photons backscattered from a 3D object. The round-trip ToF signal carries information about the depth, $z$, relative to the point of light incidence on the object's surface, which can be recovered by

$$
z=c t_{T o F} / 2
$$

where $t_{T o F}$ is the ToF of received photons, and $\mathrm{c}$ is the speed of light. The factor of two in the denominator on the right side of Eq. 1 accounts for the round-trip flight of photons.

The laser illuminates a 3D object with intensity reflectivity $R(x, y, z)$. The backscattered light signal entering the ToFCUP system, $I\left(x, y, t_{T o F}\right)$, can be linked to $R(x, y, z)$, via

$$
I\left(x, y, t_{T o F}\right)=\boldsymbol{P} R(x, y, z),
$$

where $\boldsymbol{P}$ is a lumped linear operator for light illumination and propagation. Our system then images this $3 \mathrm{D}$ object in three steps. First, the collected photons are spatially encrypted with a pseudo-random binary pattern, in which each pixel is set to either on or off. This pattern also acts as the decryption key to unlock and retrieve the image of the 3D object. Second, a streak camera temporally shears the ToF signal along the vertical direction. Third, the encrypted and sheared image is recorded on a CCD via pixel-wise spatiotemporal integration. The optical energy measured at pixel $(m, n)$ on the $\mathrm{CCD}, E(m, n)$, is related to the original 3D light intensity reflectivity, $R(x, y, z)$, by

$$
E(m, n)=\boldsymbol{T S C P R}(x, y, z) .
$$

Here, $\boldsymbol{T}, \boldsymbol{S}$, and $\boldsymbol{C}$ are linear operators that represent spatiotemporal integration, temporal shearing, and spatial encryption, respectively. Equation 3 shows that the encryption process is inherently embedded in ToF-CUP's image formation.

Image decryption can be computationally performed by users who are granted the decryption key. If the 3D object is spatiotemporally sparse, one can reasonably estimate $R(x, y, z)$ by solving the inverse problem of Eq. 3 using compressed-sensing algorithms [27-32]. In our study, we choose the Two-step Iterative Shrinkage/Thresholding (TwIST) algorithm [27], which minimizes a convex objective function given by

$$
\arg \min \left[\frac{1}{2}\|E-\boldsymbol{T S C P} R\|^{2}+\lambda \Phi_{T V}(\boldsymbol{P} R)\right] .
$$

Here, $\Phi_{T V}$ denotes the total-variation (TV) regularizer. The regularization parameter $\lambda$, which controls the weight of the TV regularizer, is adjusted empirically to provide the best estimation of $R(x, y, z)$ for a given physical reality. Further, in continuous shooting mode, the evolution of the 3D image, over the "slow time", $t_{s}, R\left(x, y, z, t_{s}\right)$, can be recovered by decrypting sequential snapshots. Here, the "slow time", $t_{s}$, relative to $t_{T o F}$, is defined as the time of capture of the imaged volume. 


\subsection{System}

The system schematic is presented in our recent publication[34]. Specifically, a solid-state pulsed laser (532 nm wavelength, 7 ps pulse duration) is the light source. The laser beam passes through an engineered diffuser and illuminates a 3D object. The object is first imaged by a camera zoom lens (focal length $18-55 \mathrm{~mm}$ ). Following the intermediate image plane, a beam splitter reflects half of the light to an external CCD camera, hereinafter called the reference camera, which records a reference $2 \mathrm{D}$ image of the $3 \mathrm{D}$ object. The other half of the light is transmitted through the beam splitter and passed to a digital micromirror device (DMD) by a 4- $f$ imaging system consisting of a tube lens and a microscope objective (focal length $45 \mathrm{~mm}$, numerical aperture 0.16 ). The total demagnification of the imaging system from the object to the DMD is $46 \times$.

To encrypt the input image, a pseudo-random binary pattern is generated by the host as the key and displayed on the DMD. Each binned pixel in the binary pattern contains $3 \times 3$ DMD pixels $(21.6 \mu \mathrm{m} \times 21.6 \mu \mathrm{m})$. The encrypted image is retro-reflected through the same 4-f system, reflected by the beam splitter, and imaged onto the fully open entrance slit ( $\sim 5 \mathrm{~mm}$ wide) of a streak camera. Deflected by a time-varying sweeping voltage, $V$, the light signal lands at various spatial locations on the $y^{\prime}$ axis according to its ToF. This temporally sheared image is recorded by a CCD camera in a single snapshot. The CCD camera has $672 \times 512$ binned pixels $(2 \times 2$ binning $)$. Finally, the encrypted image is transmitted to the user who decrypts the image with the key provided by the host.

The external CCD camera is synchronized with the streak camera for each snapshot. An USAF resolution target is used to co-register images acquired by these two devices. The reference $2 \mathrm{D}$ image is used as an intensity mask and overlaid with the reconstructed 3D image to enhance the image quality. For each snapshot, the reconstructed 3D datacube contains $N_{x} \times N_{y} \times N_{z}=150 \times 150 \times 350$ voxels along the $x, y$, and $z$ axes, respectively. In the $x-y$ plane, this size gives a maximum imaging field-of-view (FOV) of $L_{x} \times L_{y}=150 \mathrm{~mm} \times 150 \mathrm{~mm}$. The depth, $z$, can be calculated by

$$
z=n_{z} \frac{c d}{2 v}\left(1 \leq n_{z} \leq N_{z}\right),
$$

where $n_{z}$ is the pixel index along the $z$ axis, $d$ is the CCD's binned pixel size along the $y$ axis, and $v$ is the shearing velocity of the streak camera. In our experiments, $N_{z}=350, d=12.9 \mu \mathrm{m}$, and $v$ is set to $0.66 \mathrm{~mm} / \mathrm{ns}$. Therefore, the maximum depth range is $L_{z}=1050 \mathrm{~mm}$. In addition, the depth resolution of the ToF-CUP camera was quantified to be $\sim 10 \mathrm{~mm}[34]$.

\section{RESULT}

\subsection{3-D imaging of a static object}

To demonstrate ToF-CUP's 3D imaging capability, we first imaged a static object (Fig. 1). Specifically, two letters, "W" and "U", were placed at different depths, with a separation of $\sim 40 \mathrm{~mm}$ (Fig. 1a). CUP acquired an image of this 3D object in a single snapshot. The reconstructed 3D $x, y, z$ datacube of the letters "WU" is shown in Fig. 1b with the depthencoded image presented as Fig. 1c. For this object, the depth distance between these two letters was calculated to be 42 $\mathrm{mm}$, which agreed with the true value. Moreover, CUP's encryption perspective was demonstrated by comparing the image quality in the 3D datacubes reconstructed with an invalid decryption key. The reconstructed 3D datacube and the

depth-encoded image are shown as Figs. 1d and e, respectively. With the correct decryption key, the reconstructed image resembles the object. By contrast, the invalid decryption key cannot lead to any information. 


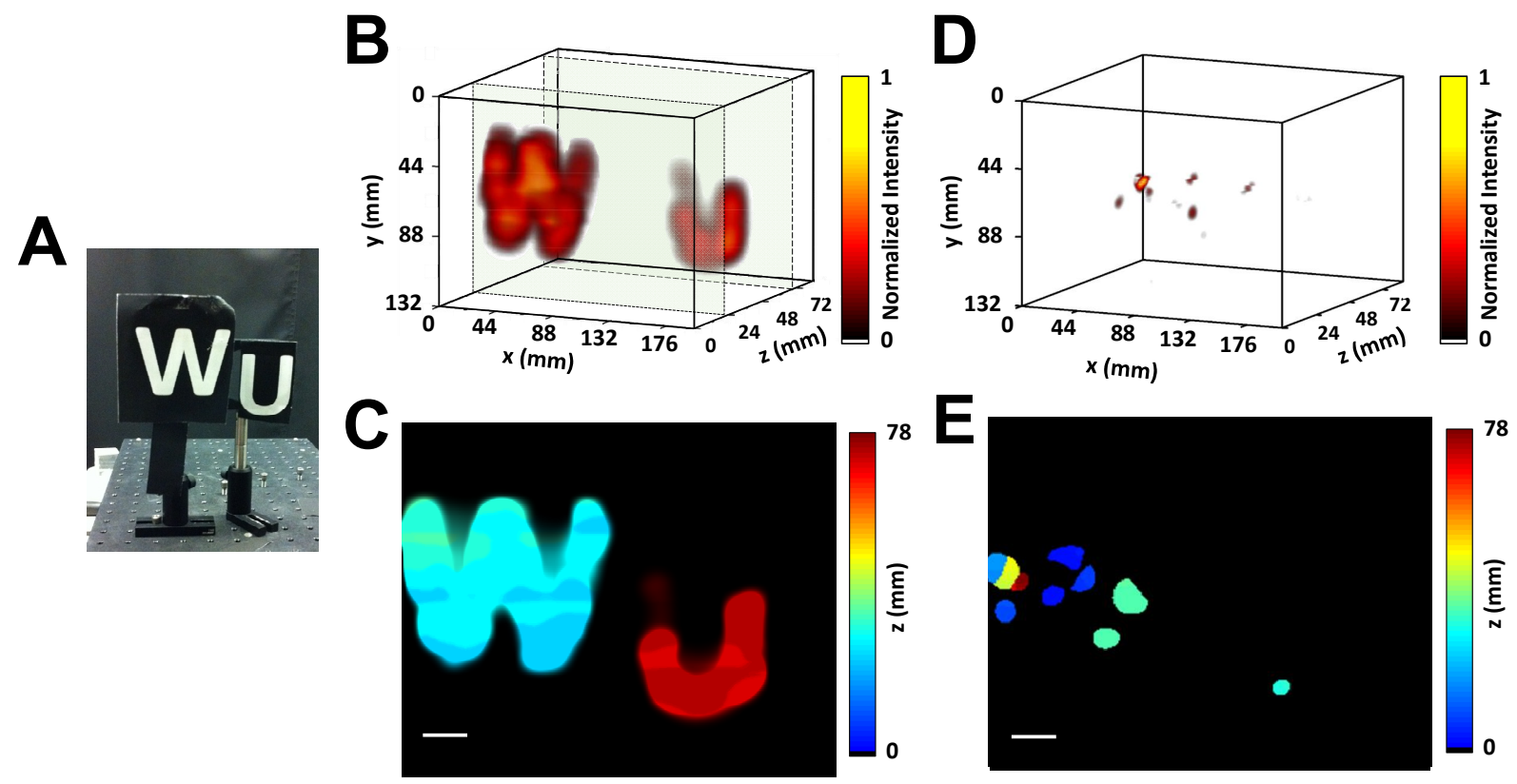

Fig. 1. ToF-CUP of a static 3D object. (a) Photograph of the letters "W" and "U", placed at different depths. (b) Reconstructed 3D $x, y, z$ datacube and (c) the depth-encoded image of letters "WU" using the correct decryption key. (d) Reconstructed 3D $x, y, z$ datacube and (e) the depth-encoded image of letters "WU" using an invalid decryption key. Scale bar: $10 \mathrm{~mm}$.

\subsection{Real-time 3-D imaging of moving objects}

To demonstrate ToF-CUP's dynamic 3D imaging capability, we imaged a rotating cube in real time (Fig. 2). In the experiment, an aluminum cube $(80 \mathrm{~mm} \times 80 \mathrm{~mm} \times 80 \mathrm{~mm})$ was rotated by a motorized stage at $\sim 70$ revolutions per minute. Two sets of patterns, a square-triangle-rhombus-circle (STRC) pattern and a heart-diamond-club-spade (HDCS) pattern, were used for imaging (Fig. 2a). CUP captured the cube's rotation by sequentially acquiring encrypted images at 24 fps. Once each encrypted image was decrypted to a 3D $x, y, z$ datacube, these datacubes form a time-lapse 4D $x, y, z$, $t_{s}$ datacube. Figures $2 \mathrm{~b}$ and $\mathrm{c}$ show representative the depth-encoded images and the $3 \mathrm{D}$ rendering of the rotating STRC and HDCS patterns at angles of $45^{\circ}$ and $20^{\circ}$, respectively. For both cases, we can successfully reconstruct the rotating motion of the 3D patterns. However, when the cube's surface rotates to a large angle with respect to the illumination, some features in the pattern cannot be reconstructed, which is attributed mainly to the low scattered light intensity. 

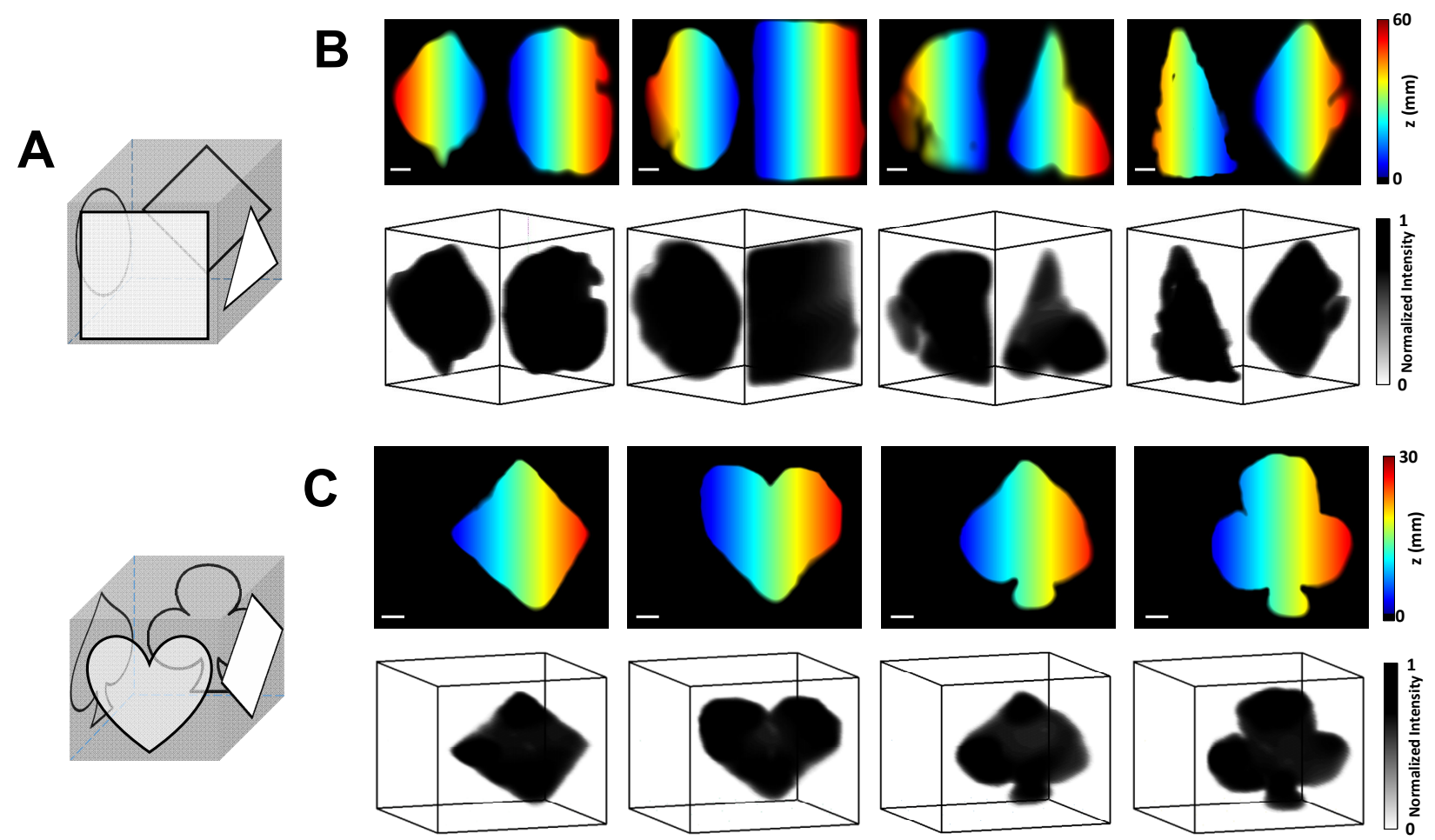

Fig. 2. ToF-CUP of a rotating 3D cube. (a) Illustrations of the two sets of patterns. Top: square, triangle, rhombus, and circle (STRC); Bottom: heart, diamond, club, and spade (HDCS). (b) Representative reconstructed depth-encoded images (top row) and 3D datacubes (bottom row) of the STRC pattern set at an angle of $45^{\circ}$. (c) Representative reconstructed depthencoded images (top row) and 3D datacubes (bottom row) of the HDCS pattern set at an angle of $20^{\circ}$. Scale bar: $10 \mathrm{~mm}$.

To apply ToF-CUP to a potential real-world application in a simulated environment, we imaged launching of a square projectile (Fig. 3). The projectile launcher is $100 \mathrm{~mm} \times 125 \mathrm{~mm} \times 100 \mathrm{~mm}$ in size along the $x, y$, and $z$ axes. The square projectile was set perpendicular to the $y$ axis $\left(\varphi=90^{\circ}\right)$ and pointed to an angle of $\theta \sim 135^{\circ}$ with respect to the $x$ axis (Fig. 3a). To capture the launching at high speed, we operated the CCD in sub-frame $(125 \times 672$ pixels $)$ mode to increase the frame rate from $24 \mathrm{fps}$ for full-frame mode to $75 \mathrm{fps}$. We first acquired a 3D image of the launcher. Then, we shifted the FOV to observe the projectile flight and acquired five snapshots within the FOV. A representative 3D image of the flying projectile in reference to its launcher is shown in Fig. 3b. The depth-encoded image of each datacube presents the projectile's trajectory (Fig. 3c), which shows a flying track approximately parallel to the $x$ axis. Top views of these datacubes comprise the projected trajectory in the $x-z$ plane (Fig. 3d), showing that the missile flew across the diagonal direction of the $x-z$ plane. The entire projectile flight can be visualized from sequential 3D datacubes (Fig. 3e). From this trajectory, the average speed of the missile was quantified to be $3.6 \mathrm{~m} / \mathrm{s}$. In addition, the projectile flew at an angle $\theta \sim 41^{\circ}$, which agrees well with the preset angle. 

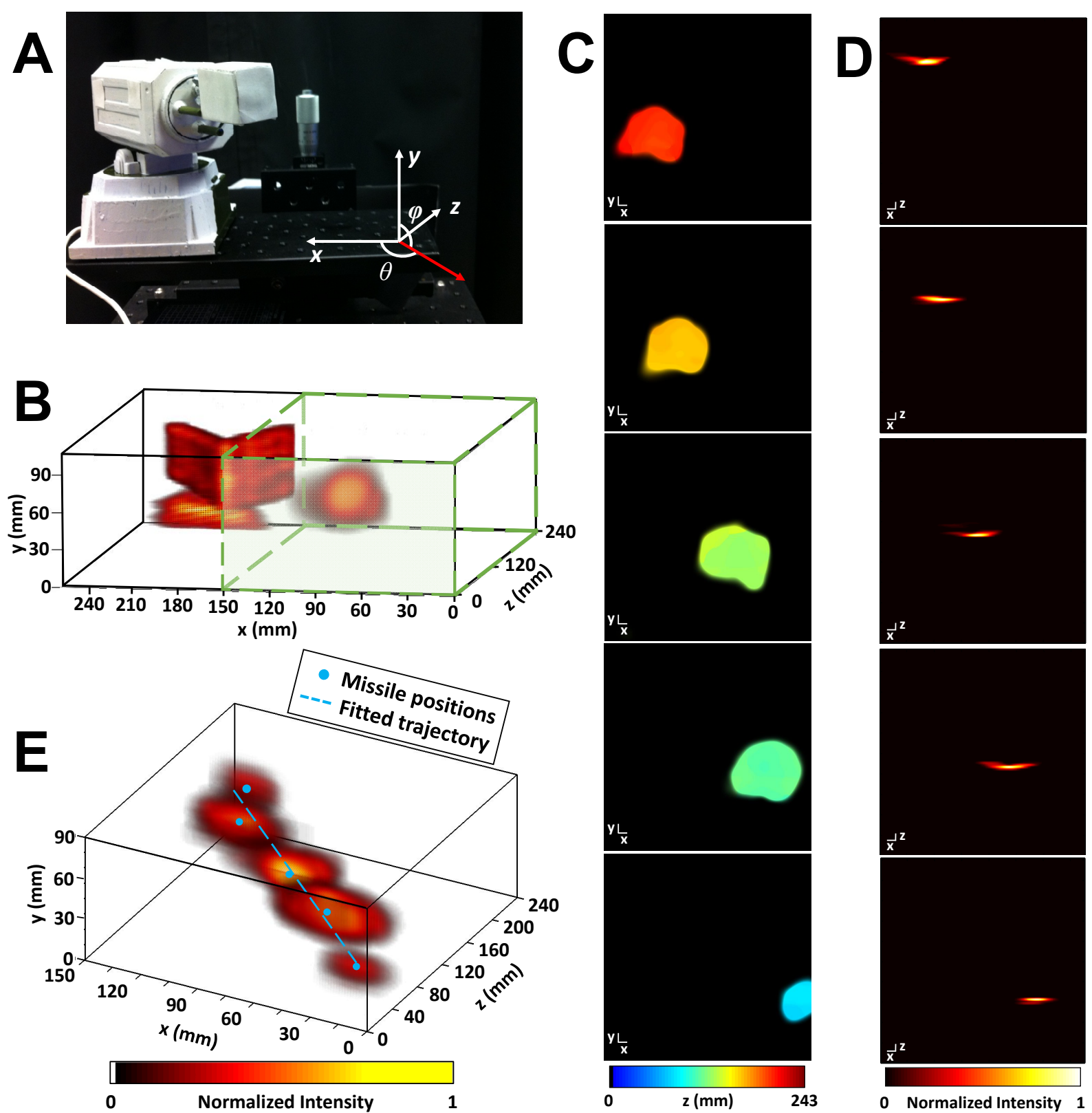

Fig. 3. ToF-CUP of a square projectile's launching and flight. (a) Photograph of the launcher. The square projectile's flight direction is marked by the red arrow in the coordinate system. (b) ToF-CUP rendering of the flying projectile at time $t_{s}=0.027 \mathrm{~s}$ in relation to the launcher. The field of view for projectile tracking is marked by the green dashed cube (c) Depthencoded front views of the flying projectile. (d) Top views of the flying projectile. (e) 3D trajectory of the flying projectile. Scale bar: $10 \mathrm{~mm}$.

\section{DISCUSSION}

Besides security, ToF-CUP offers the advantage of faster information transmission because data is compressed during acquisition. ToF-CUP compresses a 3D datacube with $N_{x} \times N_{y} \times N_{z}$ voxels to a $2 \mathrm{D}$ encrypted image with $N_{x} \times\left(N_{y}+\right.$ $\left.N_{z}-1\right)$ pixels. The compression rate can therefore be calculated as $\eta_{d}=\frac{N_{y} \times N_{z}}{\left(N_{y}+N_{z}-1\right)}$. With the current $\operatorname{setup}\left(N_{y}=\right.$ 
150 and $\left.N_{z}=350\right), \eta_{d}=105$. Therefore, CUP can potentially improve the data transmission rate by over two orders of magnitude. However, the implementation of CUP degrades the spatial resolutions by a factor of 1.8, 2.2, and 1.9 along the $x, y$, and $z$ axes, respectively, compared with the 3D image laterally limited by optical bandwidth and axially limited by the streak camera's native resolution in resolving a ToF signal[29]. Thus, regarding the actual information content, we can estimate the data compression rate by $\eta_{i}=\frac{\eta_{d}}{1.8 \times 2.2 \times 1.9}$. For the current system, $\eta_{i}=14.5$.

The ToF-CUP system's performance was mainly restricted by the illumination laser pulse energy. ToF-CUP's image reconstruction requires the DMD mask to be resolved in each encoded image at a given depth with a sufficient contrastto-noise ratio (CNR). In our experiments, the minimal value of the required CNR was empirically determined to be $\sim 8$, a value which yielded reasonable reconstruction results. To provide enough illumination intensity, the maximal FOV at the object was limited to approximately $150 \mathrm{~mm} \times 150 \mathrm{~mm}$ with the full laser energy ( $96.4 \mu \mathrm{J}$ per pulse). In addition, the maximum shearing velocity was held at $0.66 \mathrm{~mm} / \mathrm{ns}$ in the streak camera, which corresponded to a spatial sampling of 3 $\mathrm{mm}$ along the $z$ axis. With the current illumination intensity, a greater shearing velocity would reduce the number of collected photons per camera pixel and may result in an insufficient CNR.

\section{CONCLUSION}

In summary, we explored CUP's capability to image 3D dynamic objects. As a new type of ToF camera, ToF-CUP integrates image encryption, compression, and in a single snapshot measurement, significantly enhancing information safety. Our experiments demonstrated that the image of the original 3D object can be recovered by only users granted with the valid decryption key. Moreover, by leveraging sequential image acquisition, we can track a moving object in 3D and in real time. Finally, we applied ToF-CUP to image a square projectile's launching. With real-time 3D data, we recorded the projectile's trajectory and quantified the projectile's position, speed, and flight direction. To further increase the imaging FOV and depth resolution, one may utilize a high pulse-energy laser and a faster streak camera. In addition, a GPU-based reconstruction[35] should allow ToF-CUP to achieve real-time reconstruction.

\section{ACKNOWLEDGEMENT}

The authors thank Prof. James Ballard's close reading of the manuscript. This work was sponsored in part by National Institutes of Health grants DP1 EB016986 (NIH Director's Pioneer Award), R01 CA186567 (NIH Director's Transformative Research Award). L. V. W. has a financial interest in Microphotoacoustics, Inc. and Endra, Inc., which, however, did not support this work.

\section{REFERENCES}

[1] Wheatstone, C., "Contributions to the Physiology of Vision. Part the First. On Some Remarkable, and Hitherto Unobserved, Phenomena of Binocular Vision," Philosophical Transactions of the Royal Society of London, 128, 371-394 (1838).

[2] Omasa, K., Hosoi, F., and Konishi, A., "3D lidar imaging for detecting and understanding plant responses and canopy structure," Journal of Experimental Botany, 58(4), 881-898 (2007).

[3] Liu, S.-L., Li, J., Zhang, Z.-L., Wang, Z.-G., Tian, Z.-Q., Wang, G.-P., and Pang, D.-W., "Fast and High-Accuracy Localization for Three-Dimensional Single-Particle Tracking," Sci. Rep., 3, (2013).

[4] Javidi, B., Okano, F., and Son, J.-Y., [Three-Dimensional Imaging, Visualization, and Display] Springer, (2009).

[5] Koschan, A., Pollefeys, M., and Abidi, M., [3D imaging for safety and security] Springer, (2007).

[6] Bell, T., and Zhang, S., "Toward superfast three-dimensional optical metrology with digital micromirror device platforms," Opt. Eng., 53(11), 112206-112206 (2014).

[7] Kittler, J., Hilton, A., Hamouz, M., and Illingworth, J., "3D assisted face recognition: A survey of 3D imaging, modelling and recognition approachest." 114-114 (2005).

[8] Dickson, P., Li, J., Zhu, Z., Hanson, A. R., Riseman, E. M., Sabrin, H., Schultz, H., and Whitten, G., "Mosaic generation for under vehicle inspection." 251-256 (2002).

[9] Sukumar, S. R., Gorsich, D. J., Gerhart, G. R., Page, D. L., Gribok, A. V., Koschan, A. F., and Abidi, M. A., "Robotic threedimensional imaging system for under-vehicle inspection,” Journal of Electronic Imaging, 15(3), 033008-033008-11 (2006).

[10] Imaging, Z., [Deliver Mission Critical Insights] Zebra Imaging, Zebra Imaging.

[11] Trussell, C. W., "3D imaging for Army applications." 126-131.

[12] Geng, J., "Structured-light 3D surface imaging: a tutorial," Adv. Opt. Photon., 3(2), 128-160 (2011). 
[13] Huang, P. S., and Zhang, S., "Fast three-step phase-shifting algorithm,” Appl. Opt., 45(21), 5086-5091 (2006).

[14] Javidi, B., Zhang, G., and Li, J., "Encrypted optical memory using double-random phase encoding," Appl. Opt., 36(5), 1054-1058 (1997).

[15] Velten, A., Willwacher, T., Gupta, O., Veeraraghavan, A., Bawendi, M. G., and Raskar, R., "Recovering three-dimensional shape around a corner using ultrafast time-of-flight imaging," Nat Commun, 3, 745 (2012).

[16] Satat, G., Heshmat, B., Barsi, C., Raviv, D., Chen, O., Bawendi, M. G., and Raskar, R., "Locating and classifying fluorescent tags behind turbid layers using time-resolved inversion," Nat Commun, 6, (2015).

[17] Xiao, X., Javidi, B., Martinez-Corral, M., and Stern, A., "Advances in three-dimensional integral imaging: sensing, display, and applications [Invited]," Appl. Opt., 52(4), 546-560 (2013).

[18] Sun, B., Edgar, M. P., Bowman, R., Vittert, L. E., Welsh, S., Bowman, A., and Padgett, M. J., "3D Computational Imaging with Single-Pixel Detectors," Science, 340(6134), 844-847 (2013).

[19] Yi-Yuan, C., Yung-Huang, H., Yung-Cheng, C., and Yong-Sheng, C., "A 3-D surveillance system using multiple integrated cameras." 1930-1935.

[20] Miles Hansard, Seungkyu Lee, Ouk Choi, and Horaud, R. P., [Time-of-Flight Cameras: Principles, Methods and Applications] Springer, (2012).

[21] Sell, J., and O'Connor, P., "The xbox one system on a chip and kinect sensor," IEEE Micro(2), 44-53 (2014).

[22] McCarthy, A., Krichel, N. J., Gemmell, N. R., Ren, X., Tanner, M. G., Dorenbos, S. N., Zwiller, V., Hadfield, R. H., and Buller, G. S., "Kilometer-range, high resolution depth imaging via $1560 \mathrm{~nm}$ wavelength single-photon detection," Opt. Express, 21(7), 8904-8915 (2013).

[23] Medina, A., Gayá, F., and del Pozo, F., “Compact laser radar and three-dimensional camera,” J. Opt. Soc. Am. A, 23(4), 800-805 (2006).

[24] Gokturk, S. B., Yalcin, H., and Bamji, C., "A Time-Of-Flight Depth Sensor - System Description, Issues and Solutions." 35.

[25] Iddan, G. J., and Yahav, G., "Three-dimensional imaging in the studio and elsewhere." 4298, 48-55.

[26] Advanced Scientific Concepts, I., [Products overview] Advanced Scientific Concepts, Inc., Advanced Scientific Concepts(2014).

[27] Stettner, R., Bailey, H., and Richmond, R. D., "Eye-safe laser radar 3D imaging." 4377, 46-56.

[28] Orsdemir, A., Altun, H. O., Sharma, G., and Bocko, M. F., "On the security and robustness of encryption via compressed sensing." 1-7.

[29] Gao, L., Liang, J., Li, C., and Wang, L. V., "Single-shot compressed ultrafast photography at one hundred billion frames per second," Nature, 516(7529), 74-77 (2014).

[30] Shiraga, H., Nakasuji, M., Heya, M., and Miyanaga, N., "Two-dimensional sampling-image x-ray streak camera for ultrafast imaging of inertial confinement fusion plasmas," Review of Scientific Instruments, 70(1), 620-623 (1999).

[31] Heshmat, B., Satat, G., Barsi, C., and Raskar, R., "Single-shot ultrafast imaging using parallax-free alignment with a tilted lenslet array," OSA Technical Digest (online). STu3E.7.

[32] Nakagawa, K., Iwasaki, A., Oishi, Y., Horisaki, R., Tsukamoto, A., Nakamura, A., Hirosawa, K., Liao, H., Ushida, T., Goda, K., Kannari, F., and Sakuma, I., "Sequentially timed all-optical mapping photography (STAMP)," Nat Photon, 8(9), 695-700 (2014).

[33] Goda, K., Tsia, K., and Jalali, B., "Serial time-encoded amplified imaging for real-time observation of fast dynamic phenomena," Nature, 458(7242), 1145-1149 (2009).

[34] Liang, J., Gao, L., Hai, P., Li, C., and Wang, L. V., "Encrypted Three-dimensional Dynamic Imaging using Snapshot Timeof-flight Compressed Ultrafast Photography," Scientific reports, (2015).

[35] Zhu, L., Chen, Y., Liang, J., Gao, L., Ma, C., and Wang, L. V., "Space- and intensity-constrained reconstruction for compressed ultrafast photography," In review, (2016). 